\title{
Konstruktion von Autorität: Julians Hymnen ${ }^{1}$
}

\author{
Martin Hose
}

Vielleicht ist der Titel dieses Beitrags in mehrfacher Hinsicht erläuterungsbedürftig. Denn ein Hymnus ist nach geläufiger Auffassung eine Form religiöser Rede, die sich im Gestus der Anbetung ${ }^{2}$ an eine Gottheit wendet und bestimmte formale Elemente aufweist. Der Hymnus ist primär eine Kommunikationsform des Menschen mit (einem) Gott. So könnte es scheinen, dass der Begriff ,Autorität' kategorial auf einen Hymnus gar nicht anwendbar ist, da - logisch betrachtet - die Rede eines inferioren Geschöpfs gegenüber einer Gottheit aufgrund der asymmetrischen Machtverhältnisse keine Autorität entfalten kann, es sei denn, sie ergäbe sich aus der textinternen Stringenz oder der persuasiven Leistung der Rede (so etwa bei den sogenannten Zauberhymnen). Freilich entzieht sich diese Wirkung eines Hymnus auf den Gott den analytischen Möglichkeiten und dem Urteil der Philologie. Deswegen kann im Folgenden davon nicht gehandelt werden.

Stattdessen soll von anderem gesprochen werden. Die Hymnen Julians auf Helios und Magna Mater sind keine einsamen Gedanken, die ein Mensch an einen Gott richtet, sondern zu einem erheblichen Teil Darlegungen, die ein Publikum einbeziehen wollen. Sie streben an, dieses Publikum vom Dargelegten (und des-

1 Zugrunde gelegte Textausgabe: J. Bidez/G. Rochefort/Chr. Lacombrade, L'Empereur Julien. Oeuvres complètes, I.1, Paris 1932; I.2, Paris 1924; II.1 Paris 1963; II.2, Paris 1964. Ferner: Wright, W. C., The Works of the Emperor Julian, 3 Bände, London/Cambridge (Massachusetts) 1913-1923. Herangezogene Übersetzungen: Asmus, R., Kaiser Julian. Philosophische Werke, übersetzt und erklärt, Leipzig 1908. Weis, B. K., Julian. Briefe, Griechisch-Deutsch, München 1973. Goessler, L., Kaiser Julian der Abtrünnige. Die Briefe, eingeleitet, übersetzt und erläutert, Zürich/Stuttgart 1971. Folgende Arbeiten werden in abgekürzt zitiert als: Bringmann: Bringmann, Klaus, Kaiser Julian, Darmstadt 2004. Geffcken: Geffcken, Johannes, Der Ausgang des Griechisch-Römischen Heidentums, Heidelberg 1929. Marcone: Guiliano Imperatore, Alla Madre degli Dei e altri discorsi, introduzione di J. Fontaine, testo critico a cura di C. Prato, traduzione e commento di A. Marcone, Fondazione Lorenzo Valla 1987. Mau: Mau, Georg, Die Religionsphilosophie Kaiser Julians in seinen Reden auf König Helios und die Göttermutter. Mit einer Übersetzung der beiden Reden, Leipzig/Berlin 1908. Thome: Thome, Felix, Historia contra Mythos. Die Schriftauslegung Diodors von Tarsus und Theodors von Mopsuestia im Widerstreit zu Kaiser Julians und Salustius' allegorischem Mythenverständnis, Bonn 2004. Wallraff: Wallraff, Martin, Christus Verus Sol. Sonnenverehrung und Christentum in der Spätantike (Jahrbuch für Antike und Christentum, Ergänzungsband 32), Münster 2001.

2 Zur Unterscheidung von Gebet (das heißt einem dialogischen Vorgang) und Anbetung (das heißt einem monologischen Lobpreis) siehe Pulleyn, Simon, Prayer in Greek Religion, Oxford 1997, S. 55. 
sen Richtigkeit) überzeugen zu wollen. Die hierbei verwendeten rhetorischen und zumal argumentativen Strategien, die dem Text seine persuasive Dimension und damit Autorität verleihen, zu untersuchen ist Ziel dieses Beitrags. ${ }^{3}$

$\mathrm{Zu}$ Beginn ist freilich zunächst das Publikum der beiden Texte näher zu bestimmen. In der Helios-Rede scheint es durchaus nicht eindeutig, an wen sich der Text wenden will. So wird zunächst der Eindruck erweckt, Julian halte sie vor einer Festgemeinde, die die Helien am 25. Dezember in Rom begehen will, wie der Gebrauch der 1. Person Plural zeigt: $\varphi \varepsilon ́ p \varepsilon$ o ̂ิv, ö $\pi \omega \varsigma$ òv oîoi $\tau \varepsilon \hat{\omega} \mu \varepsilon v$,

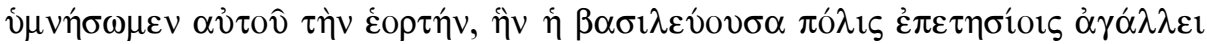
$\theta v \sigma i \alpha 15$ (131d). Jedoch darf man ausschließen, dass Julian mit seiner Rede als ,pontifex maximus' in Rom bei den Helien 362 tatsächlich auftrat, da er den Winter 362 in Antiochien verbrachte. ${ }^{4}$ Auch ist es wenig wahrscheinlich, dass dieser griechische Text nach Rom übersandt und durch Beauftragte des Kaisers vorgetragen werden sollte, da die Griechisch-Kenntnisse des Publikums nicht hinreichend gewesen wären. Damit dürfte ausgeschlossen sein, dass die Helios-Rede, wie Nilsson formulierte, eine veritable „Weihnachtspredigt“ darstellt. ${ }^{5}$ Hinzu kommt, dass im Schlussteil der Rede - ohne Vorbereitung - ein „Du“ apostrophiert wird, das der Epilog als den Vertrauten Salustios auflöst, der damit als Adressat des Textes figuriert. ${ }^{6}$ In der Rede auf die Göttermutter wird der Leser dagegen gleichsam an den Arbeitsplatz des schreibenden Kaisers geführt, wenn es heißt:

Wir haben ja nur den kleinen Teil einer Nacht ${ }^{7}$ zur Verfügung, um diese Gedanken, ohne Atem zu schöpfen, aneinander zu reihen, ohne dass wir zuvor etwas darüber nachgelesen oder darüber nachgedacht hätten; hatten wir uns doch nicht einmal vorgenommen, hierüber zu reden, bevor wir diese Schreibtafeln verlangten (178d/179a).

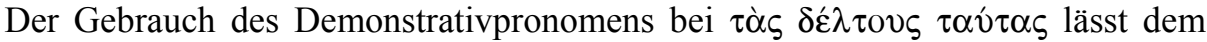
Leser in seinem Text über das Buch, das er in Händen hält, die ,Schreibtafel' des Kaisers durchscheinen.

3 So weit ich sehe, fehlen bislang spezielle Untersuchungen der rhetorischen beziehungsweise argumentativen Strategien Julians. Ausnahmen stellen dar Brox, Norbert, Gnostische Argumente bei Julian Apostata, in: Jahrbuch für Antike und Christentum 10 (1967), S. 181-186, der Julians Contra Galilaeos untersucht.; sowie Stenger, Jan, Gattungsmischung, Gattungsevokation und Gattungszitat. Julians Brief an die Athener als Beispiel, in: Würzburger Jahrbücher für die Altertumswissenschaft 30 (2006), S. 153-179, der ertragreich Julians Brief an die Athener als Beispiel für den virtuosen Umgang des Kaisers mit literarischen Formen und Formzitaten interpretiert.

4 Ammianus 22,10,1; dementsprechend halten sie Bidez (Anm. 1), S. 195, und Marcone (Anm. 1), S. 97, für in Antiochia verfasst.

5 Nilsson, Martin P., Geschichte der griechischen Religion (Band 2), München ${ }^{3} 1974$, S. 455, gebilligt von Wallraff (Anm. 1), S. 36.

6 Vergleiche Lippold, Adolf, Iulianus I (Kaiser), in: Reallexikon für Antike und Christentum 19 (2001), Sp. 442-483, hier 471.

7 Auf die Nachtarbeit des Kaisers verweisen auch Libanius, oratio 1,130, und Ammianus 16,5,4; die Helios-Rede hat Julian nach eigener Angabe (157c) in drei Nächten abgefasst. 
Beide Texte geben also keine klaren Hinweise, welchen ,Sitz im Leben' sie anstreben. Gleichwohl darf, ja muss man angesichts sowohl des Publikation (in welcher Form auch immer) erheischenden ,Kulturbetriebes' um die Mitte des vierten Jahrhunderts ${ }^{8}$ als auch des Umstandes, dass die Reden überliefert wurden, ${ }^{9}$ vermuten, dass sie Julian publiziert hat. Wahrscheinlich ist unter dieser Prämisse, dass er nicht den wohl ungewöhnlichen Publikationsweg seines Misopogon wählte, den er nach dem Zeugnis des Malalas wie ein offizielles Edikt vor dem Palast in Antiochien aushängen ließ. ${ }^{10}$ Vielmehr konnte er von seinem Text mit Hilfe der Hof-Stenographen ${ }^{11}$ und der für Antiochien bezeugten Kopisten ${ }^{12}$ eine bestimmte Anzahl von Kopien anfertigen und verschicken lassen. Über die Adressaten kann man freilich nur spekulieren; Libanios berichtet in seiner autobiographischen 1. Rede, dass der Präfekt von Antiochien einen ihm gewidmeten Panegyrikos aus der Feder des Libanios verbreiten wollte: „In der Absicht, die Rede in die wichtigsten Städte gelangen zu lassen (von da aus würde sie sich ja überall hin verbreiten), gab er zehn Schreibern einen entsprechenden Auftrag. (oratio 1,113). Die Briefe des Libanios dokumentieren im übrigen, dass rhetorische Produkte in den gebildeten Kreisen des griechischen Ostens kursierten und rezipiert wurden. ${ }^{13}$ Man kann darüber hinaus etwa aus Hinweisen im Corpus der Briefe des Synesios entnehmen, dass in der Provinz Episteln und Reden aus den kulturellen Zentren geradezu wie in Theateraufführungen vorgetragen wurden. ${ }^{14}$

Wenn nun Julian seine Reden in einer derartigen Form verbreitet und damit publiziert haben könnte, wie ist dann sein Adressatenkreis zu denken? Zwar sollte man zunächst erwarten, dass er seine beiden Traktate an ,Gesinnungsgenossen', das heißt Oreibasios, Salustios, Maximos, Priskos und andere mehr richtete, ferner an pagane Funktionsträger wie Theodoros, den Oberpriester von Asia, den nicht mehr namentlich kenntlichen Empfänger von epistula 19 (78 Hertlein), an Arsakios, den Oberpriester von Galatien (epistula 22, 49 Hertlein), an die Prieste-

$8 \mathrm{Zu}$ beachten ist ferner, dass Julian als Kaiser, im Gegensatz etwa zu Libanios (zu ihm siehe Petit, Paul, Untersuchungen über die Veröffentlichung und Verbreitung der Reden des Libanios, in: Libanios, Hg. G. Fatouros/T. Krischer, Darmstadt 1983, S. 84-128 [zuerst französisch 1956], hier 107-128), ohne Sorge um seine Sicherheit publizieren konnte.

9 Vergleiche dazu auch Russo, Carlo F., L'editore principe di Giuliano e la strategia del messagero, in: Belfagor 56 (2001), S. 437-440.

10 Malalas p. 328, 2-4 (Corpus scriptorum historiae Byzantinae, Bonn 1831), siehe dazu Wiemer, Hans-Ulrich, Ein Kaiser verspottet sich selbst: Literarische Form und historische Bedeutung von Kaiser Julians 'Misopogon', in: Imperium Romanum. Studien zu Geschichte und Rezeption. Festschrift Karl Christ, Hg. P. Kneissl/V. Losemann, Stuttgart 1998, S. 733-755, hier 736.

11 Vergleiche Libanius, oratio 18, 174.

12 Vergleiche Norman, A. F., Book Trade in Fourth-Century Antioch, in: Julian, Hg. G. Fatouros/T. Krischer, zuerst 1960, S. 267-274, hier 268/9.

13 Vergleiche etwa Libanius, epistula 27 (Fatouros, G., Krischer, T., Libanios. Briefe. Griechischdeutsch. In Auswahl herausgegeben, übersetzt und erläutert, München 1979; [= 754 Förster]).

14 Vergleiche Synesius, epistula 101, dazu Hose, Martin, Synesios und seine Briefe. Versuch der Analyse eines literarischen Entwurfs, in: Würzburger Jahrbücher für die Altertumswissenschaften 27 (2003), S. 125-141, hier 129/30. 
rin Theodora (epistulae 32-34) oder Kallixeine, die er zur Priesterin der Magna Mater in Pessinus ernannt hatte (epistula 42, 21 Hertlein). Darüber hinaus kann man vermuten, dass Julian auch an die Städte, an die er Briefe richtete, seine Reden sandte, also an die Alexandriner, die Byzankier (oder Byzanthener), die ,Thraker', die Einwohner von Bostra. Vielleicht gehörte sogar der christliche Bischof Basileios in einen solchen Adressatenkreis, zu dem Julian nach Ausweis von epistula 26 (12 Hertlein) ein freundschaftliches Verhältnis suchte, das wohl erst durch die Vorgänge um den Apollon-Tempel in Daphne Ende Oktober $362^{15}$ beiderseits unmöglich wurde.

Wenn also, zusammengefasst, die Korrespondenzpartner Julians als Parameter für den Leserkreis der beiden Reden dienen können, darf man ihren (impliziten) Adressatenkreis in der Funktionselite der griechischen Reichshälfte sehen. Sie konfrontiert Julian mit zwei programmatischen Entwürfen für eine theologisch-philosophisch fundierte Religion. ${ }^{16}$

Nach dieser Feststellung müssen zwei weitere Fragen geklärt werden: 1. Lässt sich erkennen, warum Julian gerade Helios und Magna Mater seine Reden widmet? 2. Vor welcher christlichen Polemik gegen diese Götter als Hintergrund entwickelt er seine Hymnik? Beide Fragen lassen sich, so scheint mir, beantworten. Begonnen sei mit der Helios-Rede.

Eine besondere Hinwendung Julians zu Helios ist unübersehbar. Diese Gottheit erscheint mehrfach in seinem Leben. Zosimos $(3,9,5)$ berichtet von einer nächtlichen Erscheinung und Prophezeiung des Sonnengottes, als sich Julian auf dem Zug gegen Constantius befindet; ${ }^{17}$ Libanios (oratio 18,127) bezeugt, ${ }^{18}$ dass der Kaiser im Palast in Konstantinopel ein Heiligtum für ,den Gott, der den Tag lenkt' errichtete und an dessen Mysterien teilnahm. Darüber hinaus ist Helios, ,die Sonne', natürlich traditionell für die gesamte Antike eine wichtige religiöse Bezugsgröße. ${ }^{19}$ Helios beziehungsweise Sol stellen gerade in der Spätantike eine Möglichkeit zur ,Theokrasie' dar, mittels derer Apoll, Dionysos, Hermes, Ares, Asklepios, Sarapis, Osiris, Adonis, Pan, Kronos/Saturn und Zeus als unterschiedliche Betrachtungsweisen und Verehrungsformen einer einzigen Gottheit, eben des Helios, aufgefasst werden können, wie Macrobius (Saturnalia 1,17-23) zeigt. ${ }^{20}$ Freilich hatte die Helios-Verehrung ihre besondere Geschichte, um die Julian und seine Leser genau gewusst haben dürften. Seit dem Hellenismus finden

15 Siehe dazu Lippold (Anm. 6), Sp. 462.

16 Wenn sich Julian damit als 'Religionsphilosoph' präsentiert, steht er in der Tradition Jamblichs: Siehe Smith, Andrew, Iamblichus. The First Philosopher of Religion, in: Habis 31 (2000), S. 345-353.

17 Vergleiche Ammianus 21,2,2.

18 Zum Verhältnis des Rhetors zum Kaiser vergleiche grundsätzlich Wiemer, Hans-Ulrich, Libanios und Julian. Studien zum Verhältnis von Rhetorik und Politik im vierten Jahrhundert n.Chr., München 1995.

19 Vergleiche Wallraff (Anm. 1) S. 19.

20 Vergleiche zur Theokrasie Helios-Re-Baal Iamblichus, De mysteriis 7,4/5. 
sich Formen des Sonnenkultes in verschiedensten Verschränkungen mit monarchischer Repräsentation, ${ }^{21}$ und die Geschichte des römischen Kaisertums ist durchzogen von Bestrebungen, die Bedeutung des Sonnenkultes zu steigern und für eine politische Symbolik zu nutzen. Commodus hat etwa nach Ausweis seiner Münzprägungen versucht, sein Bildnis durch eine Strahlenkrone an Sol anzugleichen. ${ }^{22}$ Die Severer waren durch ihre Affinität zum Sonnenkult in Emesa geprägt, wobei Elagabal diesen Kult sogar zum Staatskult erhob. ${ }^{23}$ Aurelian erklärte auf Münzprägungen Sol gar zum dominus imperii Romani und ließ einen Tempel für ihn errichten. ${ }^{24}$ Die Historia Augusta (in der Aurelians-Vita, einer allerdings mehr als schillernden Quelle ${ }^{25}$ ) weiß sogar zu berichten, Aurelians Mutter sei Priesterin des Sol invictus gewesen (Vita Aureliani 4,2). Der Sonnenkult war schließlich auch noch von Konstantin gepflegt worden, ${ }^{26}$ der seinen Sieg über Maxentius ${ }^{27}$ 312 auf dem sogenannten Konstantins-Bogen (geweiht 315$)^{28}$ immerhin so darstellen ließ (oder wenigstens eine solche Darstellung billigte), dass auf seinen Feldzeichen Sol unübersehbar war. ${ }^{29}$

Sol/Helios ließen sich darüber hinaus mit dem Mithras-Kult verbinden. Allerdings verzichtet Julian in der Helios-Rede auf eine solche Verbindung. ${ }^{30}$ Und schließlich ist Helios Mittelpunkt einer, Gebildeten-Religion', die aus dem Platonismus und dem Anknüpfungspunkt des Sonnengleichnisses in Platons Staat (VI

21 Siehe grundlegend Bergmann, Marianne, Die Strahlen der Herrscher. Theomorphes Herrscherbild und politische Symbolik im Hellenismus und in der römischen Kaiserzeit, Mainz 1998. Ferner Berrens, Stephan, Sonnenkult und Kaisertum von den Severern bis zu Constantin I. (193-337 n.Chr.), Stuttgart 2004.

22 Bergmann (Anm. 21), S. 265/6.

23 Bergmann (Anm. 21), S. 269-74; Wallraff (Anm. 1) S. 32/33.

24 Scriptores Historiae Augustae, Vita Aureliani 1,3; 25,6; 35,3. Siehe dazu Wissowa, Georg, Religion und Kultus der Römer, München ${ }^{2} 1912$, S. 367.

25 Zum einzelnen Halsberghe, Gaston H., The Cult of Sol Invictus, Leiden 1972, S. 131-162.

26 Siehe dazu Preger, Theodor, Konstantin-Helios, in: Hermes 36 (1901), S. 457-469; zur Rolle des Helios in Konstantinopel vergleiche Rebenich, Stefan, Vom dreizehnten Gott zum dreizehnten Apostel? Der tote Kaiser in der Spätantike, in: Zeitschrift für antikes Christentum 4 (2000), S. 300-324.

27 Der Verzicht Konstantins, nach dem Sieg zum Capitol zu gehen, um Juppiter zu opfern, scheint kein religions-, sondern ein staatspolitischer Zug gewesen zu sein, siehe Bleicken, Jochen, Constantin der Große und die Christen (Historische Zeitschrift, Beiheft 15), München 1992, S. 35; Girardet, Klaus M., Die Konstantinische Wende. Voraussetzungen und geistige Grundlagen der Religionspolitik Konstantins des Großen, Darmstadt 2006, S. 60-70.

28 Vergleiche hierzu insgesamt Raeck, Wulf, Ankunft an der Milvischen Brücke. Wort, Bild und Botschaft am Konstantinsbogen in Rom, in: $\psi v \chi \eta \dot{~-~ S e e l e ~-~ a n i m a . ~ F e s t s c h r i f t ~ K a r i n ~ A l t, ~ H g . ~ J . ~}$ Holzhausen, Stuttgart/Berlin 1998, S. 345-54.

29 Bergmann (Anm. 21), S. 283.

30 Anders unter anderem Athanassiadi-Fowden, Polymnia, Julian and Hellenism. An Intellectual Biography, Oxford 1981, S. 152/3 (Kritik dagegen bereits in der Rezension von Rosen, Klaus, in: Gnomon 55 (1983), S. 245-249); gegen einen Bezug der Helios-Rede auf den Mithras-Kult Lippold (Anm. 6) Sp. 471/2 (allerdings ohne Begründung) sowie ausführlich Smith, Rowland, Julian's Gods. Religion and philosophy in the thought and action of Julian the Apostate, London/New York 1995, S. 139-163. 
508A ff.) $)^{31}$ erwächst und die Heinrich Dörrie mit dem Begriffe der „SolarTheologie" charakterisiert hat. ${ }^{32}$

Das Christentum tat sich schwer im Umgang mit der Heliolatrie, da sowohl im Alten wie im Neuen Testament Partien mit lichtsymbolischen Aussagen eine wichtige Position einnehmen. ${ }^{33}$ Wenn etwa Christus bei Johannes $(8,12)$ sich als das „Licht der Welt“ apostrophiert und nach Maleachi $(3,20)$ als „Sonne der Gerechtigkeit" gelten soll, wenn darüber hinaus in der Kultpraxis der Osten und damit der Aufgang der Sonne bei Gebet und Kirchenbau unübersehbar war, mussten deutliche theologische Abgrenzungen zum Sonnenkult vorgenommen werden. Gleichzeitig war dem Christentum, wenn ich recht sehe, der Weg einer drastischen Polemik, wie sie etwa gegen den Apollon-Kult möglich war, ${ }^{34}$ gegen Helios versperrt: Die Sonne war und blieb Schöpfung Gottes und konnte daher nicht schlecht sein. So lassen sich folgende Strategien im Umgang mit dem Problem erkennen: Man betonte, dass die Sonne lediglich von Gott geschaffen sei. ${ }^{35}$ Ferner versuchte man, nach dem bereits bei Philon ${ }^{36}$ vorliegenden Deutungsprinzip die Rede von der Sonne beziehungsweise dem Licht allegorisch zu deuten, die Sonne als typologischen Verweis auf ihren Schöpfer zu verstehen, oder gar die Sonne analogetisch als Anschauungsobjekt zu nutzen, um durch den Vergleich mit der Sonne und ihren Strahlen das Verhältnis zwischen Gottvater und -sohn zu erläutern. $^{37}$

Dies sind - in Vereinfachung - die Kontexte, in denen Julians Helios-Rede zu sehen ist. Dass die Heliolatrie in Julians religiösem Denken eine zentrale Position innehat, zeigen die zahlreichen Verweise auf den Sonnengott in seinen Schriften. Nur hingewiesen sei etwa auf den von Julian konzipierten Mythos in der Rede gegen den Kyniker Herakleios, in dem Zeus Helios die Aufgabe zuweist, den Jüngling (= Julian) zu erziehen (oratio 7, 228d), oder die Mahnung Julians im Brief an die Alexandriner, ${ }^{38} \mathrm{zu}$ den alten Göttern und insbesondere Helios, von dessen Wirken sie lebten, zurück zu kehren:

31 Vergleiche dazu auch Ulansey, David, Mithras and the Hypercosmic Sun, in: Studies in Mithraism, Hg. J. R. Hinnells, Rom 1994, S. 259-264.

32 Dörrie, Heinrich, Die Solar-Theologie der kaiserzeitlichen Antike, in: Die Alte Kirche, Hg. H. Frohnes/U. W. Knorr (Kirchengeschichte als Missionsgeschichte 1), München 1974, S. 283292. Geprägt hat den Begriff meines Wissens Cumont, Franz, La théologie solaire du paganisme Romain, Paris 1909.

33 Zum Folgenden Wallraff (Anm. 1) S. 41-59.

34 Apollon wurde als 'böser Dämon' gedeutet, so etwa Tatian, Oratio ad Graecos 12,4; Clemens Alexandrinus, Protrepticus 2,41,2. Vergleiche Detschew, Dimiter, s.v. Apollon, in: Reallexikon für Antike und Christentum 1, Sp. 524-529.

35 So Ambrosius, Hexameron 4,2,5.

36 Philo, De somniis 1,73.

37 So bei Justin, Dialogus cum Tryphone 1.

38 Vergleiche dazu Bringmann (Anm. 1), S. 142/3. 
Seid ihr als einzige bar des Empfindens für den von Helios herniederströmenden Glanz? Wisst ihr als einzige nicht, dass durch ihn Sommer und Winter entstehen? Ist euch als einzigen unbekannt, dass alles Leben von ihm geschaffen, alle Bewegung von ihm hervorgerufen wird? [...] Und da wagt ihr es, keiner dieser Gottheiten eure Verehrung zu bezeigen, glaubt jedoch, dass Jesus, den weder ihr gesehen habt noch

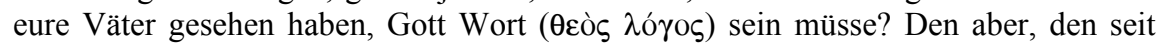
Ewigkeiten die gesamte Menschheit sieht und erblickt und verehrt und durch diese Verehrung gedeiht, den großen Helios meine ich, das lebende und beseelte, das ver-

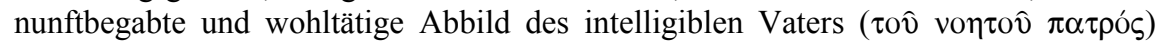
haltet ihr nicht für einen Gott? [...] (epistula 111, 434b-d).

Bereits in diesem Briefausschnitt ist die argumentative Strategie des Kaisers unübersehbar. Die eigentliche Argumentation wird über Stilmittel mit suggestiver Kraft aufgeladen: Über die drei rhetorische Fragen an die Alexandriner einleiten-

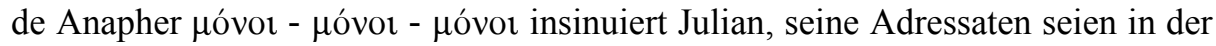
Ablehnung des Helios isoliert, ihr Christentum beruhe gar auf einem Irrtum, gegen den die ,Evidenz' des Helios stehe: Der Kaiser kumuliert Verben der Wahrnehmung, die eine ,demonstratio ad oculos` seines Beweisziels implizieren:

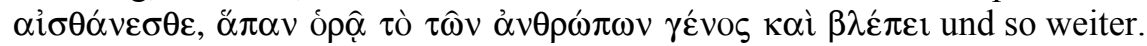

Inhaltlich fasst Julian Helios als ,sichtbaren', ja spürbar wirksamen, überzeitlichen Gott (Jahreszeiten, Bewegung) auf, er entallegorisiert ihn, wobei er zugleich eine Verbindung mit dem Neuplatonismus herstellt, indem er von einem ,intelligiblen' Vater spricht. Damit ist in nuce in diesem kurzen Textausschnitt das ,theologische Konzept“ Julians entworfen: Eine Religion, die neuplatonisch hergeleitet ist und die ihre ,Beweiskraft' aus Evidenzen der sinnlich wahrnehmbaren Welt zieht.

Komplexer und reicher begegnet dieses Konzept in der Helios-Rede. Zunächst zur Form. Wir sind es gewohnt, diese Rede als ,Hymnus‘ zu bezeichnen. Dies hat insofern seine Berechtigung, als Julian am Ende des Textes seine $\lambda$ ó $\gamma o r$

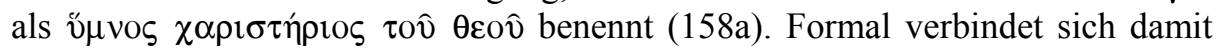
jedoch ein Problem, da die Gattung des Götterhymnos nach gerade antikem Verständnis $^{39}$ an Texte in metrischer Form (das heißt in der Regel in Hexametern) gebunden war, die ,Preislieder' für Götter mit bestimmten inhaltlichen und formalen Merkmalen darstellten und die im Kontext mit Festen für diese Götter oder mindestens kultischen Handlungen ihren ,Sitz im Leben' hatten - oder wenigstens für einen solchen konzipiert erschienen (seit dem Hellenismus ist die Möglichkeit der Ablösung von der Aufführungssituation gegeben). ${ }^{40}$ Freilich stehen die Reden Julians nicht losgelöst in der Literaturgeschichte, da seit dem zweiten

39 Siehe Färber, Hans, Die Lyrik in der Kunsttheorie der Antike, München 1936, S. 28/29 (Abhandlung); S. 26-29 (Texte; hier sind zu ergänzen: Plato, Leges III 700; Aristoteles, Ars poetica 1448b27; Athenaeus, Deipnosophistae 14,631; [Plutarch] De musica 27; Scholium in Theocritum 1,61); vergleiche ferner Harvey, A. E., The classification of Greek lyric poetry, in: Classical Quarterly 5 (1955), S. 157-175.

40 Die Literatur zum antiken Hymnos ist reich, siehe zusammenfassend Furley, William D./Fuhrer, Therese, Hymnos/Hymnus, in: Der Neue Pauly 5 (1999), Sp. 788-797. 
Jahrhundert ,Prosahymnen' nachweisbar sind. Wichtigster Vertreter dieser speziellen Hymnen-Form dürfte Aelius Aristides mit zehn (erhaltenen) hymnenartigen Reden sein (orationes $37-46$ Keil). ${ }^{41}$ Es ist nun zunächst merkwürdig, dass Julian nicht auf eine näherliegende Tradition zurückgreift, die man mit Günther Zuntz als ,philosophischen Hymnos' bezeichnen könnte, ${ }^{42}$ die etwa in Kleanthes' Zeus-Hymnos vorliegt und die Proklos aufgreifen wird. Allerdings wird Julians Wahl der Textsorte vor dem Hintergrund seines Ziels verständlich. Ein poetischer Hymnos verharrt in ,Preis' und ,Anbetung ' der Gottheit, er ist nur begrenzt persuasiv angelegt. ${ }^{43}$ An zwei Beispielen sei dies kurz erläutert. (i) In der Parodos der Bakchen des Euripides (Verse 64-169) singt der Chor ein Lied, das Elemente eines Kult-Hymnos auf Dionysos enthält, dabei aber für den Kult werben will. ${ }^{44}$ Zentrales Anliegen der Werbung und damit Ziel der Persuasion ist die Darstellung der Glückseligkeit des Dionysos-Anhängers (Verse 73 ff.: $\hat{\omega}$

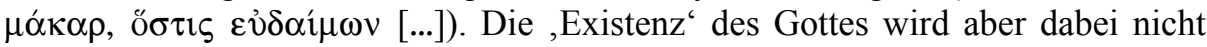
bewiesen, sondern ist vorausgesetzt. (ii) Ovid ,benutzt ${ }^{\star}$ die Form des Hymnos häufig. In den Metamorphosen etwa lässt er Apollon, der Daphne verfolgt, zu dieser eine ,Werberede' in Hymnenform vortragen (Metamorphosen 1,515-24), da er die kultische Verehrung, die ihm entgegengebracht wird, aufzählt, um mit dieser Auto-Aretalogie die Nymphe für sich zu gewinnen. ${ }^{45}$ Auch hier wird also dem (beziehungsweise der), der/die den Gott verehrt, Glück versprochen; Beweiskraft ist damit nicht verbunden - und wird auch bei Ovid nicht erreicht: Apoll bleibt erfolglos.

Der Prosa-Hymnos ist dagegen offener. Aelius Aristides gebraucht diese Form mit einem expliziten Beweisziel: Er will die Dignität der epideiktischen Rede und damit der Prosa gegenüber der Poesie etablieren (oratio 45, 1-13). ${ }^{46}$ Julians Intention beim Gebrauch dieser offeneren Form wird schlaglichtartig sichtbar, vergleicht man die Eröffnung seines Helios-Hymnos mit der des Proklischen Helios-Hymnos (Hymnus 1). Bei Proklos lautet die Eröffnung:

41 Dazu Wissmann, Jessica, Enkomion, Hymnos und Prooimion. Zu den Prosahymnen des Ailios Aristeides und Dion Chrysostomos, oratio 53, in: Studium declamatorium. Untersuchungen zu Schulübungen und Prunkreden von der Antike bis zur Neuzeit, Hg. B. J. und J.-P. Schröder, München/Leipzig 2003, S. 193-211.

42 Zuntz, Günther, Griechische philosophische Hymnen, Hg. H. Cancik/L. Käppel, Tübingen 2005, S. VIII.

$43 \mathrm{Zu}$ diesem Problem Furley, W. D., Praise and Persuasion in Greek Hymns, in: Journal of Hellenic Studies 115 (1995), S. 29-46, der zeigt, dass das persuasive Moment der Hymnen allein im Streben liegt, die Gunst der Gottheit zu erwirken.

44 Vergleiche dazu Hose, Martin, Studien zum Chor bei Euripides, Teil 2, Stuttgart/Leipzig 1991, S. $340 / 41$.

45 Hierzu Fuhrer, Therese, Der Götterhymnus als Prahlrede - Zum Spiel mit einer literarischen Form in Ovids Metamorphosen, in: Hermes 127 (1999), S. 356-67.

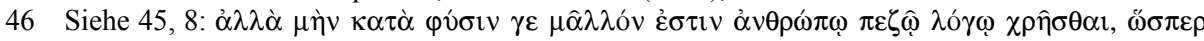

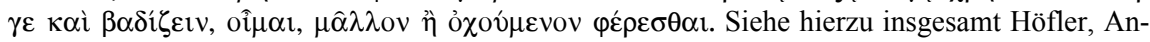
ton, Der Sarapishymnus des Ailios Aristeides, Stuttgart/Berlin 1935, S. 21-37. 


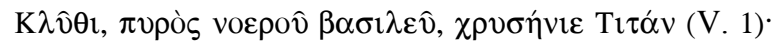

Höre, König des noerischen Feuers, Titan, der die goldenen Zügel hält [...]

Hier liegt eine konventionelle Epiklese vor, die Anrufung der Gottheit, die im Zentrum des Hymnos steht. ${ }^{47}$

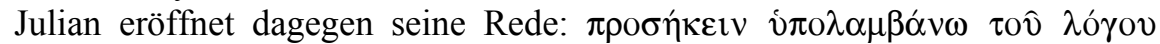

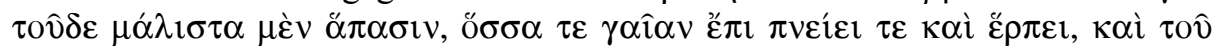

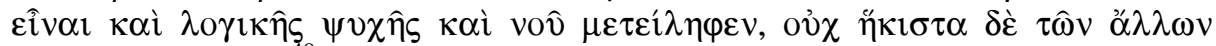

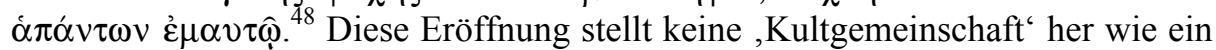
Hymnos, sondern sie behauptet (rhetorisch abgeschwächt durch die captatio benevolentiae des relativierenden $i \pi 0 \lambda \alpha \mu \beta \alpha ́ v \omega$ ) den ,Belang' der Rede für nicht etwa einfach: ,die Menschen' oder ,die Menschen, die Verstand genug haben'; vielmehr definiert Julian mit zwei Bestimmungen den Menschen, für den seine Rede relevant sein soll, in zweierlei Hinsicht. Die erste Bestimmung ő $\sigma \sigma \alpha$ ... $\pi v \varepsilon i ́ \varepsilon ı$ formuliert zwar zunächst nur den Begriff des Lebendig-Seins in einem allgemeinen Sinn, verweist aber durch die Form des Homer-Zitats $\left(\right.$ Ilias 17,447) ${ }^{49}$ auf einen Bildungszusammenhang. Stammt es doch aus einer Rede des Zeus, in der er angesichts des Todes des Patroklos und der Trauer um ihn den Menschen als das bejammernswerteste Geschöpf auf Erden bemitleidet. Mir scheint, dass der gebildete Leser diesen Zusammenhang ,mit'-hörte beziehungsweise -las. Damit trägt das Ilias-Zitat dazu bei, das $\pi \rho \circ \eta_{\kappa} \kappa \varepsilon \iota v$ des Textbeginns zu erläutern. Denn der Helios-Logos betrifft den Menschen insofern, als die dargelegte Theologie aus dem Zustand der Armseligkeit herauszuhelfen beiträgt. Denn wenn die Rede dem Rezipienten das Verstehen des Helios-Gottes erleichtert, fördert sie dessen ,Glück', da nach einem bei Jamblich (De mysteriis 10,5) formulierten

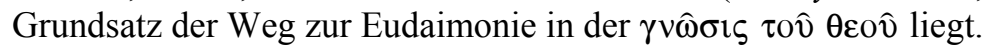

Die zweite Bestimmung, die Teilhabe an Sein, Seele und Geist, liefert in nuce die ,Anthropologie' der Rede, da über die Begriffe Sein, Seele und Geist eine neuplatonische Definition des Menschen gegeben ist. ${ }^{50}$ Zugleich wird über diese implizite Bestimmung auf die Voraussetzungen verwiesen, die gegeben sind, damit den Menschen $\gamma \nu \hat{\sigma} \sigma ı \varsigma$ $\theta \varepsilon o v ̃$ zur Eudaimonie führen kann.

An diese Eröffnung der Rede schließt Julian eine persönliche Bemerkung an,

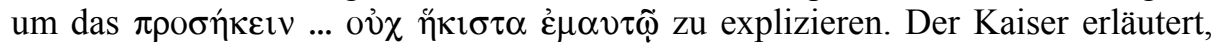

47 Zur Funktion der Hymnen des Proklos im Rahmen der Akademie siehe Erler, Michael, Interpretieren als Gottesdienst, in: Proclus et son influence, Hg. G. Boss/G. Seel, Zürich 1987, S. 179217.

48 „Dass diese Rede Belang hat, so vermute ich, am meisten für alles, was über die Erde hin atmet und kriecht, und Anteil hat am Sein, vernunftbegabter Seele und Geist, nicht am wenigsten aber für mich."

49 Zusätzlich liegt eine Reminiszenz an Kleanthes' Zeus-Hymnos, Vers 5, vor, siehe Marcone (Anm. 1), S. 291.

50 Mau (Anm. 1) S. 6-14 weist detailliert nach, dass sich Julian mit dieser Bestimmung insbeson-

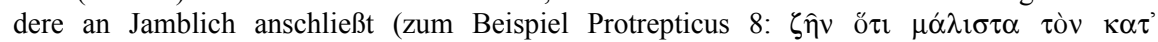

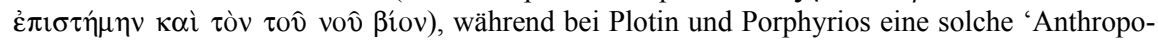
logie' noch nicht entfaltet wird. 
dass er bereits in seiner Kindheit sich unreflektiert zu den ,Strahlen des Gottes“ hingezogen fühlte. Dieses Sehnen, $\pi$ ó $\theta$ os, ist bekanntlich seit Alexander dem Großen als ,edles" Verlangen etabliert, sei jedoch bei Julian von Unwissenheit

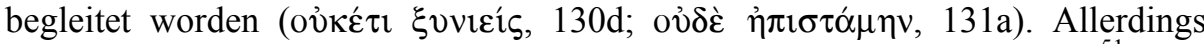
verzichtet Julian darauf, seinen eigenen Weg zum Wissen zu schildern. ${ }^{51}$ Statt

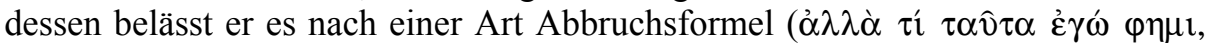
131a) bei einigen vagen Andeutungen, um nach einem Preis derjenigen, die infol-

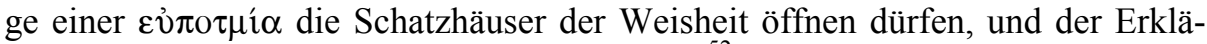

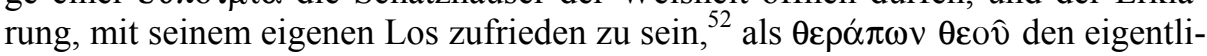
chen Preis zu beginnen ( große Teile gliedern, ${ }^{53}$ 1. die Substanz des Helios (132c-142b) und 2. seine Kräfte und Kraftäußerungen (142b-157b). Eine solche Einteilung zeigt, dass im Hauptteil der Helios-Rede in Modifikation zwei Strukturelemente der Hymnenform vorliegen, nämlich $(\sim 1$.) die Geburtslegende des Gottes und $(\sim 2$.) die Darstellung seiner Taten, die Aretalogie.

Im der Substanz (ov̉oí $\alpha$ ) gewidmeten Teil steht Julian indirekt vor der Aufgabe, einen „Gottesbeweis“ führen zu müssen. Er stellt sich dieser Aufgabe durch insbesondere zwei Argumentationsstrategien, die seiner Darlegung Plausibilität und damit Autorität geben sollen. Die eine Strategie besteht in Argumentation mit Analogien:

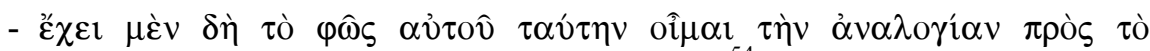

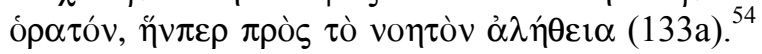

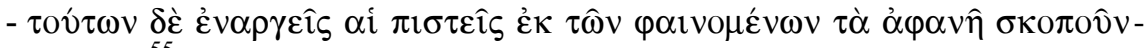
$\tau \imath(133 \mathrm{c}){ }^{55}$

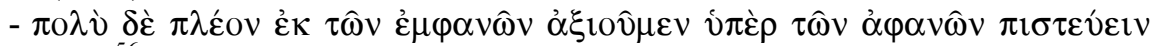
$(138 \mathrm{~b}){ }^{56}$

Julian verwendet die Analogie als Argumentationsmittel in verschiedenen Formen, um ausgehend vom sinnlich Wahrnehmbaren auf Unsichtbares zu schließen. Dabei ,springt' er durchaus auch zwischen Bereichen, die nicht zusammengehören, etwa, wenn er nicht nur von der Reinheit des Lichts auf die Lauterkeit des Helios schließt (140d), sondern sogar die formende Funktion des Lichts, die Voraussetzung der Wahrnehmung von Gegenständen sei, mit der Arbeit und For-

51 In welchem Maß hier eine Selbststilisierung (und keine autobiographische Nachricht) vorliegt, kann außer Betracht bleiben, vergleiche zum Problem Rosen, Klaus, Kaiser Julian auf dem Weg vom Christentum zum Heidentum, in: Jahrbuch für Antike und Christentum 40 (1997), S. 126146.

52 Zur Partie 131c siehe Marcone (Anm. 1), S. 293.

53 Vergleiche Asmus (Anm. 1), S. 133/34. Julian selbst gibt diese Disposition in 132b vor.

54 „Es steht nun, glaube ich, sein Licht in eben dem Verhältnis zum Sichtbaren wie die Wahrheit zum Intelligiblen."

55 „Die Beweise hierfür ergeben sich für den, der das Unsichtbare erforscht, aus dem Sichtbaren.“

56 „Vielmehr halten wir es für angemessen, auf der Grundlage des Sichtbaren über das Unsichtbare Beweisgründe (respektive: Glauben) zu haben.“ 
mung des Goldschmieds an einer Bildsäule plausibel machen will (134c/d). Mit dieser Verwendung der analogetischen Argumentation greift er auf die alte griechische naturphilosophische Methodologie zurück, die unter dem Schlagwort

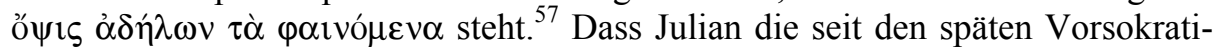
kern greifbare Skepsis gegenüber der sinnlichen Wahrnehmung ignoriert, sei am Rande festgehalten. ${ }^{58}$

Die zweite Argumentationsstrategie liegt darin, sich auf Autoritäten zu stützen (wobei Julian allerdings auf, Altersbeweise' verzichtet ${ }^{59}$ ). Platon (Politeia VI 508b: 132d; 133a), Homer (Ilias 8,840; Odyssee 1,80: 136d; Ilias 8,24: 137a; Ilias 18,239/40; 21,6/7: 137b), Hesiod (Theogonie 371-74: 136c), Aristoteles (De caelo II 12, 291b34: 138a/b), Empedokles (B 27/28 Diels-Kranz: 138d) werden namentlich $^{60}$ aufgeführt, um bestimmte Aussagen über die Stellung des Helios zu den anderen Göttern zu belegen. Hinzu kommt ein Vers, der nicht zu identifizieren ist. Er soll für Julian die Theokrasie belegen:

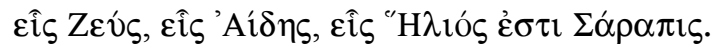

Julian deklariert diesen Vers als Orakel Apolls (135a/136a). Da bei Macrobius

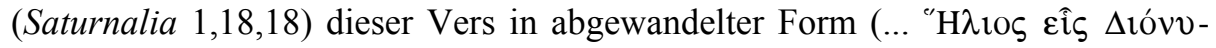
$\sigma o \varsigma)$ als orphisch zitiert ist, stützt sich Julian, so die communis opinio, hier eigentlich auf Jamblich, der aber nicht ausdrücklich genannt ist. ${ }^{61}$

Gestützt auf diese beiden Argumentationsstrategien entwirft Julian ein komplexes und bisweilen kompliziertes Bild der ov̉oí $\alpha$ des Helios, das damit nicht idiosynkratisch, das heißt aus neuplatonischen Axiomen, sondern aus auch für „Nicht-Neuplatoniker“ nachvollziehbaren Argumenten hergeleitet erscheint. Die-

57 Dazu Regenbogen, Otto, Eine Forschungsmethode antiker Naturwissenschaft, in: Kleine Schrif-

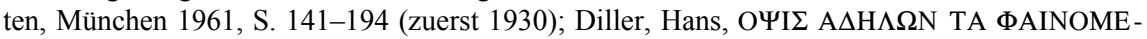
NA, in: Kleine Schriften zur antiken Literatur, München 1971, S. 119-143 (zuerst 1932).

58 Dazu Lloyd, G. E. R., Magic, Reason and Experience, Cambridge 1979, S. 133-36.

59 Anders zum Beispiel Plutarch im Amatorius 13 (Moralia 756b): ,[...] weil du für jeden einzelnen Gott Rechtfertigung und Existenznachweis verlangst. Da muss die von den Vätern ererbte alte Überzeugung genügen; über sie hinaus kann niemand einen klaren Beweis geben und entdecken [...]“ (Übersetzung Herwig Görgemanns). Zur Rolle der Altersargumentation im Platonismus siehe Erler, Michael, Legitimation und Projektion: Die 'Weisheit der Alten' im Platonismus der Spätantike, in: Die Gegenwart des Altertums, Hg. D. Kuhn/H. Stahl, Heidelberg 2001, S. 313-326.

60 Darüber hinaus lassen sich im Text zahlreiche weitere, nicht als Zitat ausgewiesene Anklänge an Platon erkennen, die Marcone (Anm. 1), passim, nachgewiesen hat.

61 Siehe Geffcken (Anm. 1), S. 105 mit Anm. 11; Kern, O. (coll.), Orphicorum Fragmenta, Berlin 1922, Nummer 239. Zur latenten Benutzung von insbesondere Jamblichs (verlorenem) Kommentar zu Platons Alkibiades siehe Asmus, R., Der Alkibiades-Kommentar des Jamblichos als Hauptquelle für Kaiser Julian (Sitzungsberichte der Heidelberger Akademie der Wissenschaften), Heidelberg 1917, Heft 3. Die sicher bestimmbaren Fragmente (siehe Dillon, J. M., Iamblichi Chalcidensis in Platonis Dialogos Commentariorum fragmenta, Leiden 1973, dort S. 72-83) geben jedoch keine deutlichen Hinweise. Dillon (S. 13) hat daher auch erwogen, Jamblichs (ebenfalls verlorener) Schrift Über die Götter einen maßgeblichen Einfluss zuzuschreiben. 
ses Bild kann er katechismusartig ${ }^{62}$ zusammenfassen (141a-142a). Der Befund der Argumentationstechnik für diesen ,Helios-Gottesbeweis' scheint nun zunächst unspektakulär. Aufschlussreicher wird er freilich, wenn man zugleich bedenkt, was Julian hier nicht tut: Er verzichtet darauf, das neuplatonische System der Welt als ein einfach gegebenes zu referieren (Die apriori-Setzung des $\check{\varepsilon} v$ war ja der entscheidende Schritt für Plotin ${ }^{63}$ ). Er verzichtet auf den Gebrauch der Chaldäischen Orakel, er verzichtet auf Referenztexte des Neuplatonismus wie Jamblich - jedenfalls als Bausteine der Argumentation. In bedeutsamer Weise hebt er sich sogar implizit gegen Porphyrios und Jamblich ${ }^{64}$ ab. Denn wenn Julian Helios zum Mittelpunkt der Welt macht, nimmt er eine Position ein, die Jamblich wie Proklos ausdrücklich ablehnten und statt Helios die $\psi v \chi \eta \dot{~ i m ~ Z e n t r u m ~ d e r ~}$ Welt verankerten. ${ }^{65}$ Julian gebraucht dagegen die ,Klassiker ${ }^{6}$ und eine allgemein akzeptierte Methode. Jeder Gebildete könnte daher die Argumentation nachvollziehen. Ich komme darauf zurück.

Der längere, zweite Teil der Rede ist den Fähigkeiten und Kräften des Helios gewidmet, die in drei Schritten gewürdigt werden. Julian handelt zuerst von den

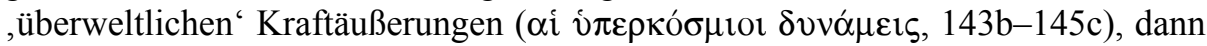
den Kraftäußerungen in der sichtbaren Welt, die sich am Himmel vollziehen ( $\alpha i$ $\pi \rho \circ \sigma \kappa o ́ \sigma \mu ı \imath ~ \delta v v \alpha ́ \mu \varepsilon เ \varsigma, 145 \mathrm{c}-150 \mathrm{~d})$, und schließlich den Kraftäußerungen ,unter dem Monde', das heißt in der Welt (150d-157b).

Im ersten Schritt bedient sich Julian in der Darstellung der $\dot{\pi \varepsilon \rho \kappa o ́ \sigma \mu t o r ~}$ $\delta v v \alpha ́ \mu \varepsilon ı \varsigma$ wiederum der Analogie, um die Kraft des Helios aufzuweisen, mit der

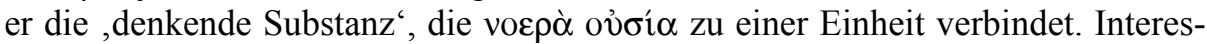
santerweise gebraucht er dabei nicht allein die Physik, das heißt zieht die Gesamtverbindung der Grundelemente zu einem Kosmos heran (143c), sondern auch die Ästhetik. Denn Helios ist qua Theokrasie auch Apollon Musagetes, der (scili-

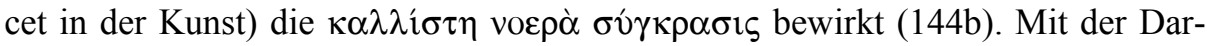
stellung der Kräfte in der Welt hat es Julian leichter, da er nunmehr die ,Evidenz',

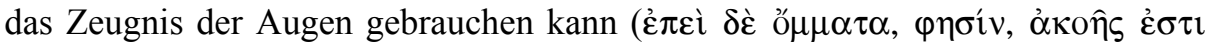
$\pi \_\tau \tau$ ó $\varepsilon \rho \alpha, 145 \mathrm{c}$ ). Diese ,Evidenz', etwa des Sternenhimmels (vergleiche 146d:

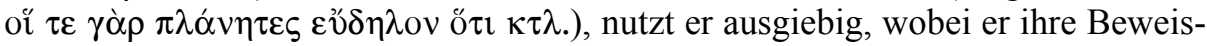
kraft durch den Hinweis erhöht, dass seine Beobachtungen und Schlüsse von allen

62 Gallinari, A., Il pensiero politico-educativo dell' imperatore Giuliano l'Apostata, Cassino 1995, S. 107, verweist auf die Nähe der sprachlichen Gestaltung dieser Partie zum Nicaenum.

63 Zusammenfassend dazu Dörrie, H., Plotin. Philosoph und Theologe, in: Platonica Minora, München 1976, S. 361-374 (zuerst 1963).

64 Vergleiche Bregman, Jay, Elements of the Emperor Julian's Theology, in: Traditions of Platonism. Essays in Honour of J. Dillon, Hg. J. J. Cleary, Aldershot 1999, S. 337-350, hier 343, Anm. 15.

65 So Proclus In Platonis Timaeum 171d, vergleiche Mau (Anm. 1) S. 74/75. Siehe auch Witt, R. E., Iamblichus as a forerunner of Julian, in: De Jamblique a Proclus, Hg. H. Dörrie (Entretiens sur l'antiquité classique 21), Vandoeuvres-Genève 1974, S. 35-64, hier 53/4, der die andere Rolle des Helios bei Julian auf dessen Mithras-Verehrung zurückführt. Meine Überlegungen versuchen die Differenz mit der persuasiven Funktion zu erklären. 
nachvollzogen werden können, „die den Himmel nicht bloß beiläufig oder wie Hornvieh betrachten“ (148c).

Die Schöpfung lässt mithin die Menschen die sie wirkenden Götter und hinter

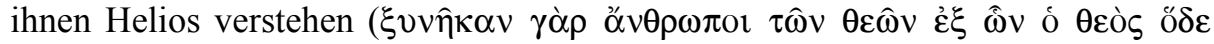

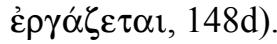

Lediglich für eine durchaus kühne Konstruktion muss Julian ein anderes Beweisverfahren heranziehen: Abweichend vom Mythos will er Athene nicht aus dem Haupt des Zeus, sondern aus König Helios als ganzem geboren sein lassen (149b). Hierfür zieht er zwei Hexameter heran, von denen der zweite aus Homer stammt (Ilias 8, 540), der erste nicht identifizierbar ist. ${ }^{66}$ Ein kurzer Exkurs verstärkt Julians Konzept des Götterhimmels durch einen ,Altersbeweis': Über eine von Jamblich ${ }^{67}$ stammende interpretatio Graeca der in Emesa gemeinsam mit Helios verehrten Gottheiten Monimos und Azizos als Hermes und Ares gewinnt der Kaiser ein weiteres Indiz für die von ihm postulierte Position des Helios unter den Göttern.

Die weitere Argumentation über Helios' Wirkungen in der sichtbaren Welt fokussiert auf dessen, Wohltaten'. Julian berührt dabei die Vorstellung vom Aufstieg der Seelen nur in einer Praeteritio ${ }^{68}$ und stuft sie als Gegebenheit ein, die „mehr geglaubt als dargestellt“ (= bewiesen) werden könne $(\pi \imath \sigma \tau \varepsilon v \varepsilon \dot{\sigma} \sigma \theta \omega \mu \hat{\alpha} \lambda \lambda$ ov خे $\delta \varepsilon \imath \kappa v v ́ \sigma \theta \omega, 152 b)$. Stattdessen stellt er wiederum die ,Evidenz', das für alle

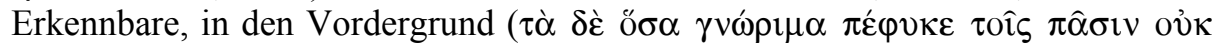

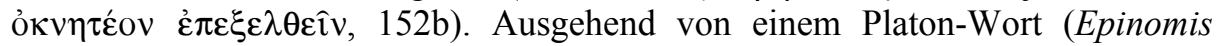

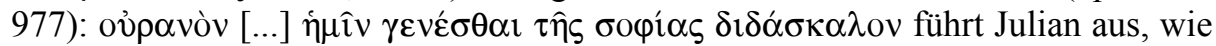
der Mensch durch Helios sowie die von ihm ausgehenden Götter Dionysos und Apoll seine Weisheit, seine ,Kultur' und seine staatliche Ordnung gewonnen habe. ${ }^{69}$ Mit dem Gedanken an die Kultivierung der Welt durch griechische Gesittung und römische Herrschaft (152d) gelingt es Julian, einen, gleitenden Übergang' zu Rom (das er antiquarisch als griechische Gründung deutet) und den Helien herzustellen, die der Ausgangspunkt der Rede waren. ${ }^{70}$ Allerdings frappiert hier die Argumentation, da Julian alles daran setzt, auch Rom als Produkt

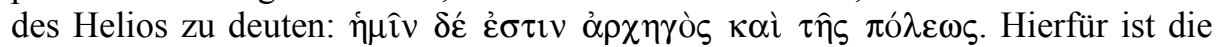
Theokrasie Voraussetzung, die Helios mit Zeus/Juppiter (auf dem Capitol) und

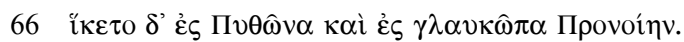

67 Es ist nicht zu ermitteln, ob die für Jamblich bezeugte Junktur von Hermes und Ares mit Helios mit der bei Macrobius (Saturnalia 1,19,1/7) für Jamblich zu gewinnenden Information über dessen Identifikation von Hermes, Ares und Helios zu verbinden ist.

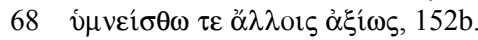

69 Hier begründet Julian wiederum sein Bild der Rolle des Helios per analogiam: Dass Helios 'Aphrodite und Athene' einsetzte und Sexualität lediglich als Mittel der Fortpflanzung aufzufassen befehle, werde durch den von Helios vollzogenen Jahresverlauf gezeigt, der alle Lebewesen (suo tempore) zur Reproduktion veranlasse.

70 Vergleiche Classen, Carl-Joachim, Julian und die Römer, in: Würzburger Jahrbücher für die Altertumswissenschaften 26 (2002), S. 151-170, hier 164/5. 
mit Apoll (auf dem Palatin) gleichsetzt (153d). ${ }^{71}$ Interessant ist der Weg, auf dem Julian die die Zwillinge säugende Wölfin mit Helios verbindet: ${ }^{72}$ Da das Jahr auf

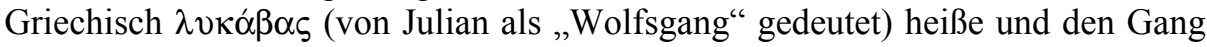
der Sonne/des Helios bezeichne, sei erwiesen, dass der Wolf Helios heilig sei. Romulus/Quirinus wird auch auf Helios bezogen, indem die Sage über seinen Tod durch Blitzschlag ${ }^{73}$ als Vernichtung lediglich des Sterblichen an ihm und Indiz für den Wiederaufstieg seiner Seele zu Helios durch Hilfe Selenes gelesen wird (154c/d). Als abschließenden Beweis für die Bedeutung des Helios führt Julian die Kalender- und Festbräuche anderer Völker und schließlich der Römer an.

Der Kaiser beendet seine Rede mit einem Gebet an Helios und die anderen ,königlichen Götter'. In diesem Gebet, das zunächst eine lange Reihe von Appositionen enthält, die das Wesen des Helios apostrophieren und damit die Epiklesen des Hymnos repräsentieren, bittet Julian ,seinen' Gott, seinen Dienst für das Reich leisten zu dürfen.

Damit könnte die Rede schließen. Doch Julian fährt mit einer Apostrophe an Salustios fort. Das gerade Dargelegte habe der Kaiser in drei Nächten verfasst.

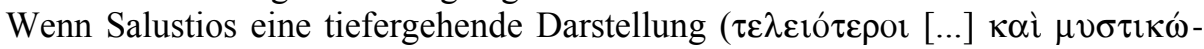
$\tau \varepsilon \rho o \imath ~ \lambda o ́ \gamma o r, 157 \mathrm{c})$ wolle, müsse er die entsprechenden Schriften Jamblichs lesen, die die Vollendung menschlicher Weisheit darstellten ( $\tau$ ò $\tau \hat{\varepsilon} \lambda \mathrm{\lambda}$ [ [...] $\tau \hat{\eta} \varsigma$

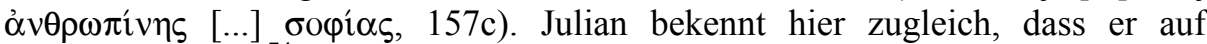
Jamblich aufbaut. ${ }^{74}$ Diese Verbeugung vor Jamblich ist zwar angesichts der Bedeutung von dessen Platonismus für den Kaiser nicht überraschend, ${ }^{75}$ in ihrer Zuspitzung, die letztlich die gesamte Schrift entwerten müsste, nähme man sie beim Wort, jedoch zunächst erstaunlich. Hinzu kommt, dass Julian, soweit erkennbar, ${ }^{76}$ deutlich von Jamblich abweicht. Denn der Kaiser hat nicht vov̂ $\psi v \chi \eta ́$ ins Zentrum der Welt gestellt, sondern Helios.

Diese Merkwürdigkeiten müssen erklärt werden. Am Anfang der Rede hatte Julian die Bedeutung dessen, was er sagen würde, auch für sich selbst festgestellt. Ferner hatte er darauf verzichtet, im Referat seiner Beziehung zu Helios von Kindesbeinen an seinen eigenen Weg vom Ahnen zum Wissen auszuführen. Am

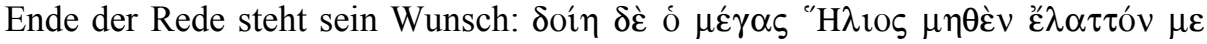

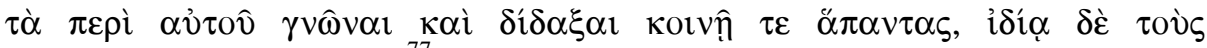
$\mu \alpha \nu \theta \alpha ́ v \varepsilon ı v ~ \alpha ̉ \xi i ́ o v \varsigma$ (157d). ${ }^{77}$ Es scheint, dass damit noch einmal auf den Anfang der Rede zurückgegriffen wird. Allerdings ist durch die Rede selbst ein $\gamma v \hat{\omega} v \alpha \imath$

71 Vergleiche Mau (Anm. 1), S. 87.

72 Vergleiche damit Macrobius, Saturnalia 1,17,39.

73 Vergleiche Plutarch, Vita Romuli 12,2.

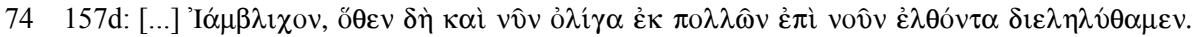

75 Vergleiche etwa auch $146 \mathrm{~b}$.

76 Vergleiche Mau (Anm. 1), S. 38/39.

77 „Möge mir aber der gewaltige Helios vergönnen, ebenso zur Erkenntnis seines Wesens zu gelangen und alle insgesamt, ganz besonders aber diejenigen, die es kennen zu lernen verdienen, darüber zu belehren.“ 
$\tau \grave{\alpha} \pi \varepsilon \rho \grave{~} \mathrm{H} H \lambda$ íov für den Leser möglich und idealiter vollzogen. Doch nicht nur für den Leser, auch für den auctor Julian selbst, der mit seiner Argumentation geradezu seinen Weg vom Ahnen zum Wissen nachvollzieht. Der Text, so darf man behaupten, stellt eine argumentative Selbstvergewisserung des Autors dar. Dieser Form der argumentativen Selbstvergewisserung, die nicht einfach die ,Lehre“ Jamblichs referiert, sondern mit Beweisgängen operiert, ist auch die systemische Abweichung geschuldet. Hätte Julian den vov̂s ins Zentrum seiner neuplatonischen Welt gestellt, wäre seiner Argumentation die ,Evidenz', auf deren Beweiskraft er, wie gezeigt, so nachhaltig setzt, verloren. Heinrich Dörrie hat den Neuplatonismus als Theologie einmal so charakterisiert: „Diese Theologie schließt keine Heilslehre und noch weniger eine Heilserwartung ein. Sie bildet vor allem keine Gemeinde; sondern jedem ist es überlassen, selbst den Weg zur Erkenntnis zu finden. ${ }^{678}$ Julian aber, will' die Gemeinde, er braucht sie für seine ,Kirche'. Er kann es daher nicht einfach den Menschen (wobei er sich natürlich, wie eingangs dargelegt, nicht den ,Massen', sondern den intellektuellen Eliten zuwendet) überlassen, selbst zur $\gamma \nu \hat{\omega} \sigma \iota \varsigma \theta \varepsilon o \hat{v}$ zu gelangen. Er will sie dezidiert für seine Sache gewinnen (ein Neuplatoniker stünde diesem Ziel fremd gegenüber), anleiten, belehren und überzeugen. Dafür braucht er beweiskräftige Instanzen. Jamblichs vôิ würde keine Überzeugungskraft haben, da eine Jamblichsche Theologie keinen Gottesbeweis mit Indizien aus der sichtbaren Welt ermöglicht. So weicht Julian von Jamblich ab und errichtet ein System um einen auch sichtbaren, das

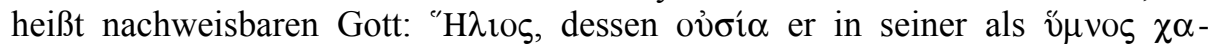

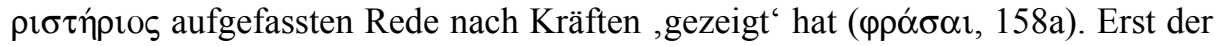
so für eine neuplatonische Theologie Gewonnene wird auf Jamblich und dessen ,tiefere' Einsicht verwiesen.

Anders als die Helios-Rede, die dem ,Beweis' des Gottes gewidmet war, steht Julian in der Rede für die Göttermutter vor einem doppelten argumentativen Problem. Nicht allein hatte er die christliche Polemik gegen Kybele und ihren Kult zu entkräften, vielmehr genügte es nicht, der Tradition, das heißt einem ekstatischen und sinnlichen Kult aufzuhelfen. Julian musste das Bild der Göttermutter seinen grundsätzlichen Vorstellungen vom Göttlichen und ihren Kult seiner Vorstellung von der ,Reinheit ${ }^{79}$ auch der Priester ${ }^{80}$ anpassen. ${ }^{81}$

78 Dörrie, Heinrich, Plotin. Philosoph und Theologe, in: Platonica minora, München 1976, S. 36174, hier 372.

79 Vergleiche dazu Bowersock, Glen Warren, Julian the Apostate, London 1978, S. 79-93: „The Puritanical Pagan“.

$80 \mathrm{Zu}$ den politischen Dimensionen dieser Erwartungen an Priester vergleiche Brauch, Thomas, The Political Philosophy of the Emperor Julian as found in his Writings, Administration and Propaganda, Ph.D.-Thesis, University of Minnesota 1980, S. 277-284.

81 Vergleiche zu diesem Problem insgesamt Cosi, Dario M., Casta Mater Idaea. Giuliano l'Apostata e l'etica della sessualità, Venedig 1986, wobei allerdings eine zum Teil biographische Betrachtungsweise (La vita sessuale di Giuliano, S. 60-75) vorliegt. 
Der Kybele-Kult war ein leichtes Ziel für christliche Angriffe. ${ }^{82}$ Die Kultpraktiken mit ihrer speziellen Verbindung von Blut und Sexualität figurieren seit den Apologeten (Aristides, Apologie 11,5; Justin, Apologie 1,27,4 und öfter) als Monument von Unsittlichkeit und Grausamkeit. Man spottet über einen ,kastrierten Gott, man ,euhemerisiert" die Kultlegende von Kybele und Attis zu einer Geschichte einer eifersüchtigen Königin (Firmicus Maternus, De errore 3). Kurz und prägnant benennt das unter dem Namen Cyprians überlieferte Carmen Ad quendam senatorem ex Christiana religione ad idolorum servitutem conversum den Kult als Brandmal, das eine infamia turpis in seine Anhänger einbrennt (Vers 8). ${ }^{83}$

Dass der Kybele-Kult um die Mitte des vierten Jahrhunderts Anziehungskraft besaß, ${ }^{84}$ zumal in seiner Ausgestaltung der Riten und Mysterien Momente einer Heilserwartung und Erlösung sichtbar waren, zeigt etwa der sogenannte Festkalender des Filocalus von 354, der für den Zeitraum vom 15. bis zum 28. März einen liturgischen Ablauf zeigt, der mit einer Schilfprozession und einem Stieropfer beginnt, das an Attis' Schicksal am Fluss Gallos erinnern soll, Fastentage enthält ( $\vee \eta \sigma \tau \varepsilon i \alpha$, Sallust, De diis 4) und mit geheimen Zeremonien endet. ${ }^{85}$ Festkalender und das pseudo-cyprianische Carmen zeigen, dass Julian einen durchaus nicht erstorbenen Kult ${ }^{86}$ fördern wollte, dass seine Maßnahmen ${ }^{87}$ dem Hauptort des Kybele-Kultes, Pessinus, den er wohl im Sommer 362 besucht hatte, ${ }^{88}$ wieder zu altem Glanz zu verhelfen, nicht apriori hoffnungslos waren.

Allerdings gab es ein Problem. Die am orgiastischen Kybele-Kult Beteiligten, zumal die Priester, repräsentierten das durch Kybele und Attis dargestellte Prinzip von Tod und Wiedergeburt ekstatisch und sexuell konnotiert. Julian verlangte von

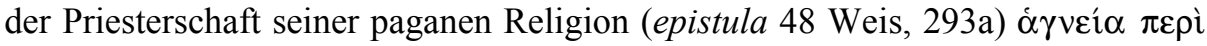

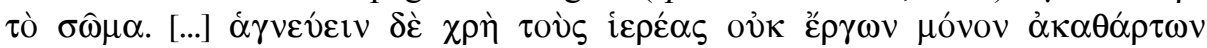

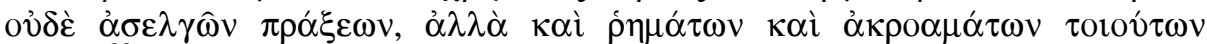
(300c). ${ }^{89}$ Er verbietet ihnen sogar die Lektüre von Archilochos und Hipponax

82 Siehe hierzu Ristow, Günter, Kybele, in: Reallexikon für Antike und Christentum (im Druck).

83 Vergleiche dazu Nock, A. D., Conversion. The Old and the New in Religion from Alexander the Great to Augustine of Hippo, Oxford 1933, S. 159/60.

84 Noch immer wichtig dazu Bloch, Herbert, The Pagan Revival in the West at the End of the Fourth Century, in: The Conflict between Paganism and Christianity in the Fourth Century, Hg. A. Momigliano, Oxford 1963, S. 192-218, hier besonders 202-204.

85 Vergleiche Thome (Anm. 1), S. 52.

86 Vergleiche die Nachweise bei Geffcken (Anm. 1), S. 294/95.

87 In einem Brief (epistula 36 Weis, 388c) ernennt er eine Demeter-Priesterin, Kallixeine, zusätzlich zur Priesterin der Kybele in Pessinus; in einem Brief an Asarkios, den Oberpriester von Ga-

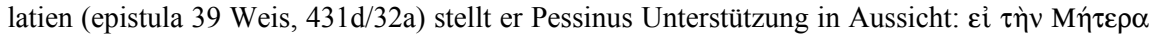

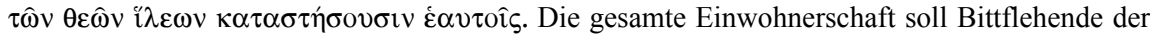
Göttin werden.

88 Anders Bringmann (Anm. 1), S. 120: „In Pessinus erlebte er die bittere Enttäuschung, dass der Kult und die Verehrung der Göttin fast in Vergessenheit geraten war."

89 „Rein halten müssen sich aber die Priester nicht nur von unreinen Werken und zuchtlosen Handlungen, sondern auch von ihrem Aussprechen und Anhören.“(Übersetzung Weis). 
(300c). Unter dieser Prämisse ist es erstaunlich, dass Julian einen Hymnos, das heißt wiederum eine Lobrede auf die Göttermutter verfasste.

Die Methode, die Julian für die Rettung des Kybele-Kultes wählte, ist die Allegorese. Er bediente sich damit eines Interpretationsverfahrens, das eine bis ins fünfte Jahrhundert v.Chr. zurückreichende Tradition hatte und sich etwa bei der Verteidigung der religiösen Valenz des Homer-Textes bewährt hatte. ${ }^{90}$ Auch die jüdische Theologie hatte durch Aristobulos und Philon entsprechende Versuche unternommen, exegetische Probleme in Teilen der Tora allegorisch zu bewältigen. Hier lag auch ein Ansatzpunkt für antichristliche Polemik: Kelsos, so jedenfalls kann man einer - leider kurzen - Notiz bei Origenes (Contra Celsum 4,51) entnehmen, kritisierte jüdische und christliche Versuche der Allegorese, dass diese noch , schändlicher und absurder' als die zu erklärenden Mythen seien ( $\tau \hat{\omega} v$

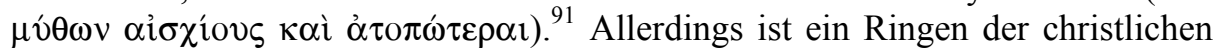
Theologie um das exegetische Prinzip der Allegorese unübersehbar. Origenes hatte versucht, Kelsos' Spott über die Genesis („Märchen für alte Frauen“, 4,39) durch Analogisierung dieser Teile der Bibel mit Platons Mythen zu begegnen, dafür aber die harsche Kritik der christlichen Theologen geerntet, die auf einem streng literalen Verständnis der Bibel beharrten. ${ }^{92}$ Zugleich wollte man sich aber dieses Instruments nicht ganz begeben, wie etwa Augustin zeigt, der zwar in De Genesi contra Manichaeos die Manichäer der Willkür in der Textauslegung durch Allegorese beschuldigt ${ }^{93}$ und den Literalsinn verteidigt, in De doctrina christiana $(3,10,14)$ jedoch reklamiert: ut quidquid in sermone divino neque ad morum honestatem neque ad fidei veritatem proprie referri potest, figuratum esse cognoscas.

Dass Augustin hier eine Maxime formuliert, die auch in paganen Kulten praktiziert wurde, zeigt eine Notiz in einem Brief, in der er berichtet, wie er Zeuge wurde, wie in paganen Tempeln Priester dem Volk Göttermythen allegorisch auslegten. ${ }^{94}$ Julian würde Augustin gewiss zugestimmt haben: At enim illa omnia, quae antiquitus de vita deorum moribusque conscripta sunt, longe aliter sunt intellegenda atque interpretanda sapientibus.

90 Vergleiche Hammerstaedt, Jürgen, Die Homerallegorese des älteren Metrodor von Lampsakos, in: Zeitschrift für Papyrologie und Epigraphik 121 (1998), S. 28-32, mit weiterer Literatur.

91 Hierzu Cook, John G., The Interpretation of the Old Testament in Greco-Roman Paganism, Tübingen 2004, S. 63.

92 Vergleiche etwa Eustathius von Antiochien, De engastrimytho contra Origenem 21,3; 22; Theophilus von Alexandria, In Esaiam 6,1-7; Theodor von Mopsuestia, Commentarius in epistulam ad Galatos 4,24. Dazu insgesamt Fiedrowicz, Michael, Prinzipien der Schriftauslegung in der Alten Kirche, Bern/Berlin/Frankfurt(Main)/New York/Paris/Wien 1998.

93 Siehe Pollmann, Karla, Doctrina Christiana. Untersuchungen zu den Anfängen der christlichen Hermeneutik unter besonderer Berücksichtigung von Augustinus, De doctrina christiana, Freiburg/Schweiz 1996, S. 18/19.

94 Epistula 91,5, dazu Nock (Anm. 83), S. 159. 
Hiermit konvergiert Julians Position, ${ }^{95}$ dass gerade das Seltsame, Absurde der Mythen den Weg zur Wahrheit bahne: ,Je auffallender und wunderbarer eben das Rätsel ist, um so eindringlicher scheint es uns zu beschwören, nicht den bloßen Worten zu glauben, sondern uns um das verborgene Geheimnis zu bemühen [...]", schreibt er in seiner Rede gegen den Kyniker Heraklios (217c) und wiederholt den Gedanken in der Rede auf die Göttermutter (170a). ${ }^{96}$

Das Ergebnis einer solchen Bemühung um das verborgene Geheimnis stellt

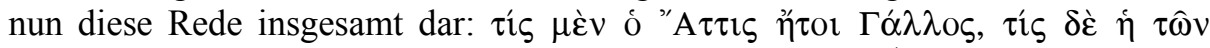

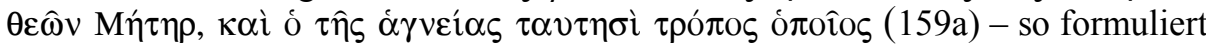
Julian zum Eingang der Rede die Aufgabenstellung des Rätsels, das er im Folgenden lösen wird. Dabei geht er radikaler als andere Interpreten vor, da er nicht nur den Mythos selbst allegorisiert, sondern auch den Kult. Letzteres ist freilich der Notwendigkeit geschuldet, $\alpha \gamma v \varepsilon i ́ \alpha$ auch den Priestern zuschreiben zu können, die buchstäblich unsittlich handeln.

Die Argumentation der Rede besteht mithin in einer großen Allegorese, einer Interpretation, die Kybele und Attis als Wesenheiten der intellektuellen Welt auffasst, in Attis die Substanz des zeugenden und schöpferischen Geistes ( $\tau 0 \hat{v}$

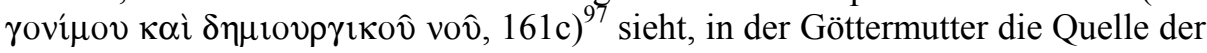

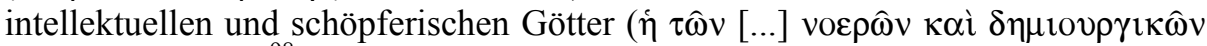
$\theta \varepsilon \hat{\omega} \vee \pi \eta \gamma \eta \dot{n}, 166 \mathrm{a})^{98}$ und schließlich im Fluss Gallos die Kreisbahn der Milchstraße (165c). ${ }^{99}$ Der Mythos, in dem Kybele aus Eifersucht Attis entmannt, mutiert dementsprechend bei Julian zur verhüllten Beschreibung eines kosmologischen Geschehens, nach dem das Prinzip der Schöpfung und des Werdens (= Attis) in die Materie hinabsteigt, sich zum Sein umwendet (das heißt hier zur Mutter) und in seiner schöpferischen Bewegung innehält (das heißt hier: Entmannung). ${ }^{100}$

Um diese Deutung mit Autorität zu versehen, bedient sich Julian verschiedener Mittel. Zunächst operiert er mit dem Gesichtspunkt der ,Tradition'. Er referiert die Einführung des Magna-Mater-Kultes in Athen (159a) und in Rom (159c) und leitet daraus den anerkannten Wert des Kultes und damit der Göttin ab (160d: $\check{\varepsilon} \delta \varepsilon 1 \xi \varepsilon v$ [ein Verbum, das den ,Beweis‘ anzeigt] $\dot{\eta} \theta \varepsilon o ́ \varsigma[\ldots])$. Ferner interpretiert

95 Siehe dazu Thome (Anm. 1), S. 39-42. Vergleiche auch Riedweg, Christoph, Mythos mit geheimem Sinn oder reine Blasphemie? Julian über die mosaische Erzählung vom Sündenfall

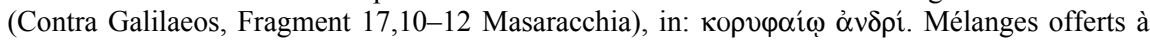
André Hurst, Hg. A. Kolde/A. Lukinovich/A.-L. Rey, Genf 2005, S. 367-75, der instruktiv an einem Beispiel die Position Julians zur allegorischen Deutung des Alten Testaments untersucht.

„Die Alten suchten vielmehr stets die Gründe der Dinge unter Anleitung der Götter [...] sie suchten sie unter Führung der Götter zu finden und [...] hüllten sie das Resultat in seltsame Mythen, damit wir durch die Seltsamkeit und das Widersprechende die Erdichtung entdecken und dadurch zum Aufsuchen der Wahrheit angeregt werden sollten."

97 Mau (Anm. 1), S. 92/93 bezieht dies auf Jamblichs 'Dritten nus'.

98 Dazu Mau (Anm. 1), S. 100/1.

99 So auch Salustius 4, Mau (Anm. 1), S. 98/99.

100 Vergleiche 175a/b. 


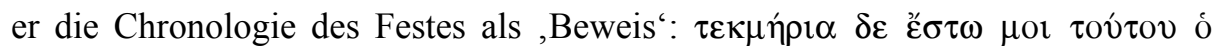

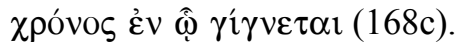

Die Beweiskraft und Autorität der Rede ergibt sich damit aus der Schlüssigkeit der Allegorese und den Indizienbeweisen aus Tradition und Chronologie. Hierzu gehört ferner, dass Julian durch einen Trick die Stringenz seiner allegorischen Deutung behauptet. Denn für den Anspruch auf Geltung seiner Interpretation ist nahezu entscheidend, dass es keine alternativen Deutungen von Mythos und Kult gibt, da sonst die Autorität des Vorgetragenen durch die mit der Alternative vorgenommene Relativierung zusammenbräche. Eine solche Bedrohung für Julians Deutung lag indes vor. Denn Porphyrios hatte, wie Eusebios bezeugt, den Attis als Symbol für den Frühling gedeutet. ${ }^{101}$ Diese Auffassung wäre hinreichend verhängnisvoll für Julians Allegorese. Dies erklärt eine seltsame Partie der Rede auf die Göttermutter. Julian schreibt (162c): „Über den Gegenstand [...] hat, wie ich höre, auch der Philosoph Porphyrios schon geschrieben. Da ich jedoch seine Auffassung nicht gelesen habe, so weiß ich freilich nicht, ob meine Rede gelegentlich einmal in irgend einem Punkt mit ihr zusammentreffen wird." Julian kann Porphyrios' Traktat nicht gänzlich ignorieren, erspart sich aber über die Fiktion der Unkenntnis eine Auseinandersetzung, die für seine Konstruktion fatal wäre.

Man hat Julians Götterhymnen gelegentlich kritisch beurteilt. Es habe ihm an der Zeit für eine sorgfältige Vorbereitung gefehlt, seine Gedankenführung sei flüchtig und die Texte deswegen schwer verständlich. ${ }^{102}$ Dass diese Vorwürfe zutreffen, wird jeder Leser bestätigen. Gleichwohl darf man den Reden eine argumentative Kraft attestieren, die das Ziel Julians, eine pagane Theologie zu konstruieren, fördern. Die Neuplatoniker späterer Zeit hat Julian beeindruckt. Es scheint, dass Proklos' Hymnendichtung auf Helios (Hymnus 1) und die Göttermutter (Hymnus 6) auch Julian rezipierte. ${ }^{103}$ Bezeichnend ist, dass Marinos in seiner Proklos-Biographie den Tod seines Lehrers nach der Regierungszeit Julians datiert. ${ }^{104}$

101 Eusebius, Praeparatio evangelica 3,11,12. Vergleiche Mau (Anm. 1), S. 92 mit Anmerkung 4.

102 Bringmann (Anm. 1), S. 117.

103 Vergleiche dazu Van den Berg, Robert M., Proclus' Hymns. Essays, Translations, Commentary, Leiden/Boston/Köln 2001, S. 170 und 172.

104 Marinus, Vita Procli 36. 
